(67\%) reported securing large furniture items in place within their home. Many parents were aware of the hazards highlighted (average 1-10 scale rating, 8.2), and found the campaign useful (average 1-10 scale rating, 7.3). In 50\% of cases the potential to alter current practices perceived by carers was encouraging (average $1-10$ scale rating, 5.3).

Conclusion A poster campaign highlighting hazards implicated in local deaths is deemed useful by parents. Further work through the use of focus groups and parental communication is required to identify how best to promote safety practices for future campaigns.

\section{G459(P) IMPROVING THE INVESTIGATION OF UNEXPECTED INFANT DEATHS}

1) Garstang, ${ }^{2} \mathrm{C}$ Ellis, ${ }^{3} \mathrm{~F}$ Griffiths, ${ }^{1} \mathrm{P}$ Sidebotham. ${ }^{1}$ Division of Mental Health and Wellbeing, University of Warwick, Warwick, UK; ${ }^{2}$ Faculty of Health and Life Sciences, Coventry University, UK; ${ }^{3}$ Division of Health Sciences, University of Warwick, Warwick, UK

\subsection{6/archdischild-2015-308599.413}

Aims Since 2008, in England, all unexpected infant deaths must be investigated jointly by police, health and social services. This study aims to learn of bereaved parents' and professionals' experiences of this joint agency approach (JAA) as well as assess the effectiveness of the JAA in determining causes and risk factors for deaths and use this knowledge to improve professional practice.

Methods

1. A mixed methods study of JAA investigation of SUDI cases in one English region; involving case note analysis, questionnaires and in-depth interviews with bereaved parents and the relevant professionals.

2. A descriptive study of outcomes of JAA SUDI investigation using Child Death Overview Panel (CDOP) data.

Results 23/111 families were recruited giving theoretical saturation. The median time between infants' deaths and parental study participation was 33 weeks; data collection took place between 2011-3. 25 professionals were interviewed. CDOP Form Cs were obtained for 65/70 (93\%) SUDI cases dying during 2010-2.

Non-specialist police often arrived at the parents' home along with the ambulance; increasing parental distress.

Parents felt that the JAA provided information about why their baby died but offered minimal emotional support.

The joint home visit by police and paediatrician is a key investigative process and most parents found this helpful. Final case discussions were used to discuss relevant risk factors but not to determine the cause of death; in nearly all cases the final cause of death relied on post-mortem examination alone ignoring findings of death scene examinations. Many deaths fitted the diagnostic criteria for SIDS but despite this were labelled as unascertained.

Social care were only involved in 13/23 JAA investigations, in two cases without involvement there were safeguarding concerns.

Some Coroners were reluctant to share post-mortem examination reports with paediatricians preventing effective JAA investigations.

Conclusion Ideally, SUDI investigations should be carried out only by specialist clinicians who do this work frequently and the JAA fully integrated with social care and Coroners' investigations. There needs to be a clearer system for classifying unexplained SUDI. Police should reconsider their immediate response to SUDI; parents would like more follow-up and bereavement support from professionals.

\section{G460(P) NEUROCOGNITIVE DEFICITS AND COMORBIDITIES IN NEUROFIBROMATOSIS1 - EXPERIENCE FROM A TERTIARY SERVICE}

R Mithyantha, H Royden, J Acharya, Z Bassi. Department of Community Paediatrics, Alderhey Children's Hospital NHS Foundation Trust, Liverpool, UK

\subsection{6/archdischild-2015-308599.414}

Aims Neurofibromatosis type $1(\mathrm{NF} 1)$ is a common genetic condition with multisystem involvement. Clinical presentation of this condition can be varied, requiring clinicians to be aware of associated comorbidities and complications. We relate our experience of children seen within a tertiary centre.

Methods We analysed 100 consecutive patients with an established diagnosis of NF1 seen in a tertiary NF1 clinic. Records of patients were reviewed to determine clinical presentation and associated range of comorbidities.

Results Of the 100 patients (M:F $=55: 45$; median age 11.6 years (range 9 months-21 years)), 57 had at least one neurodevelopmental concern ranging from Learning difficulties $32 \%$, specific learning difficulties $2 \%$, social communication difficulties including ASD in 12\%, attention difficulties including ADHD 18\%, significant behavioural difficulties 11\%, coordination difficulties $8 \%$, sleep problems $13 \%$, anxiety and low self esteem $3 \%$, epilepsy in $4 \%$. Majority of these children were in mainstream schools, so appropriate support would need to be provided for.

$7 / 100(7 \%)$ of patients developed intracranial tumours, $14 \%$ optic pathway gliomas, 8 had non-tumour cranial abnormality identified on imaging. 10 children required neurosurgical intervention. $30 \%$ of children developed scoliosis, 5/30 required surgical fixation. 4 children had pseudoarthrosis, including a late presentation at 10 years. $17 \%$ had refractory errors. $14 \%$ of children underwent investigations for poor growth, 2 required hormone replacement therapy. 6 patients underwent investigations for concerns relating to puberty ( 2 precocious puberty, 2 premature adrenarche, 2 delayed puberty). 3 patients were on treatment for hypertension (Renal artery stenosis 2, essential hypertension 1). Plexiform neurofibromas, which can cause pain and affect cosmetic appearance, were present in $27 / 100$ patients (9 required surgery).

Conclusions Under recognition of associated neurocognitive deficits and neuropsychiatric conditions in children with a diagnosis on NF1, can have significant impact on their educational outcome. The varied spectrum of clinical presentation warrants a comprehensive assessment for this group of patients. Establishing networks between local services and specialist centres will assist in early identification and management of Neurofibromatosis1 related complications.

\section{G461(P) CHARACTERISTICS OF HAEMOPHAGOCYTIC LYMPHOHISTIOCYTOSIS AT A TERITIARY PAEDIATRIC CENTRE}

F Welsh, R Hague. Royal Hospital for Sick Children, NHS Greater Glasgow and Clyde, Glasgow, UK

10.1136/archdischild-2015-308599.415 
Aim Haemophagocytic lymphohistiocytosis (HLH) is a rare, lifethreatening disorder. It can present to many specialities and individual Paediatricians are unlikely to develop significant experience of it. Early recognition and treatment improves the clinical outcome. The genetic basis of HLH is evolving and identification of viral triggers influencing management.

We aimed to study cases of HLH presenting to our tertiary Paediatric Hospital to identify the proportion of de novo cases and evaluate the role of Ebstein Barr virus (EBV) and genetic defects in the pathogenesis of HLH.

Methods Immunology, Rheumatology and Haematology Consultants identified children diagnosed with HLH over a 10 year period from 2003-2013. ICD10 codes were cross referenced. Case notes and electronic data were interrogated to extract relevant data.

Results 20 children were diagnosed with HLH over the 10 year period. Seven patients were de novo diagnoses and 13 had known haematological malignancy or rheumatological conditions.

At presentation, 15\% met the HLH 2004 diagnostic criteria, rising to $45 \%$ at time of diagnosis (Figure 1).

Viral triggers were investigated by EBV PCR and/or throat swab in 50\% of patients and all de novo HLH patients were EBV positive. Of those found to be EBV positive, Rituximab was given to 4 patients. Genetic mutations were looked for in $40 \%$ of all patients (Figure 2).

Nine patients recovered with standard management, 6 died and 5 proceeded to bone marrow transplant.

Conclusions HLH can present to a variety of paediatric specialities and a high index of suspicion is needed to make a timely diagnosis, as not all patients have 'classical' features at presentation. Identification of EBV gives the opportunity to use Rituximab effectively. In children with unusual responses to ubiquitous viruses, such as EBV, the competency of the host should be questioned. Some genetic defects predisposing to HLH in whom long term management with stem cell transplant can be curative are already known, and further defects may be discovered in the future. Accurate genetic diagnosis not only influences management, but is important for the families affected.

\section{G462(P) CAPTURING T-CELL RECEPTORS. A POTENTIAL NEW MODALITY FOR TARGETING HEPATIC TUMOURS AND POST-TRANSPLANTATION LYMPHOPROLIFERATIVE DISEASE (PTLD)}

${ }^{1,2} \mathrm{ND}$ Ruth, 'S Penny, 'L Steadman, 'D Millar, ${ }^{3} \mathrm{D}$ Hunt, ${ }^{3} \mathrm{P}$ Trantham, ${ }^{1,2} \mathrm{D}$ Kelly, ${ }^{1} \mathrm{M}$ Cobbold. 'School of Infection and Immunity, University of Birmingham Medical School, Birmingham, UK; ${ }^{2}$ Hepatology, Birmingham Children's Hospital NHS Foundation Trust, Birmingham, UK; ${ }^{3}$ Department of Chemistry, University of Virginia, USA

\subsection{6/archdischild-2015-308599.416}

Background The immune system plays an important part in recognising infection and diseases including cancer. Malignant cells express specific proteins on their cell surface. It is widely believed that it is these proteins that the immune system uses to recognise tumours and eventually eradicate them. When this process goes wrong, a tumour forms. The immune response to cancers in general is widely accepted to play an important role

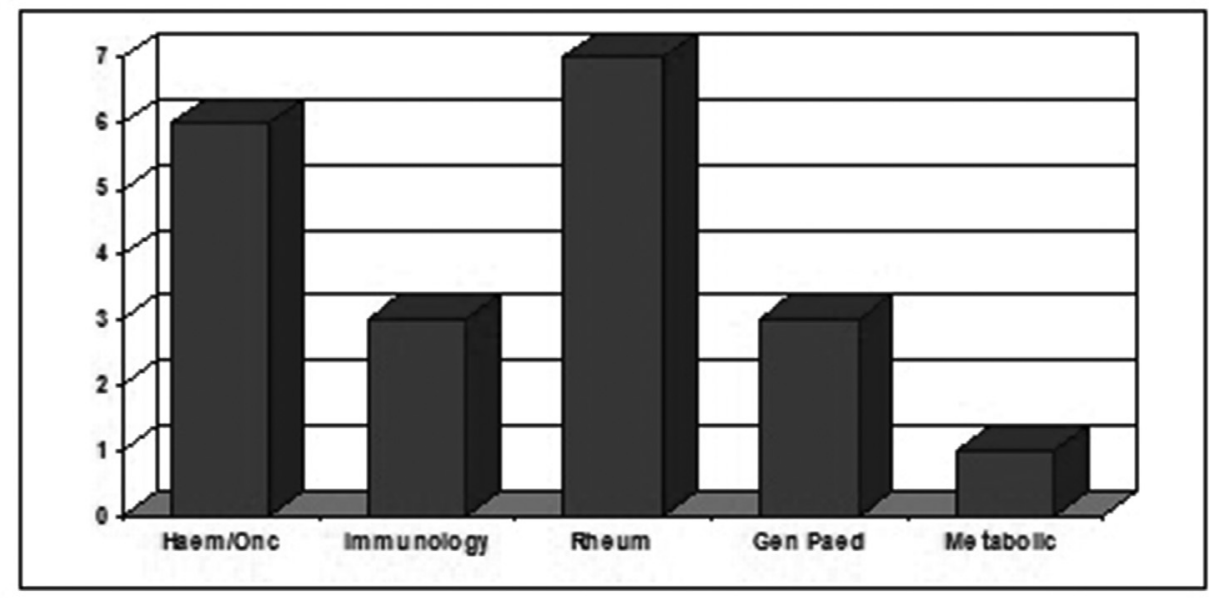

Abstract G461(P) Figure 1 Presenting specialities of children diagnosed with HLH

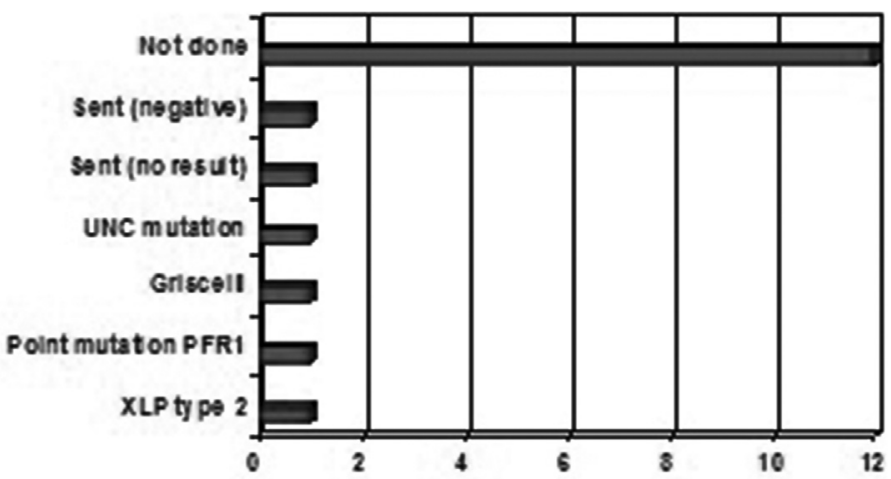

Abstract G461(P) Figure 2 Genetic studies in children with HLH 